\title{
Sensorless Speed Control of Permanent Magnet Synchronous Motor by Unscented Kalman Filter using Various Scaling Parameters
}

\author{
Cheol Moon* and Young Ahn Kwon ${ }^{\dagger}$
}

\begin{abstract}
This paper investigates the application, design and implementation of unscented Kalman filter observer using the various scaling parameters for the sensorless speed control of a permanent magnet synchronous motor. The principles of unscented transformation and unscented Kalman filter are examined and their applications are explained. Typically the mapping transformation process is divided into two types, namely the basic unscented transformation and the general unscented transformation by virtue of the scaling parameter value. And resultantly, the number of sampling points, weights, code configuration and computation time are different. But there is no little information on the scaling parameter value or how this value influences the system performance. To analyze the unscented transformation with the various scaling parameters in this study, the experimental results under a wide range of operation condition have been demonstrated.
\end{abstract}

Keywords: Unscented Kalman filter, Unscented transformation, Scaling parameter, Sensorless speed control, Permanent-magnet synchronous motor

\section{Introduction}

A permanent-magnet synchronous motor (PMSM) has the advantages of high efficiency and excellent torque characteristics comparing with other electric machines. These advantages enable PMSM to be widely applied in industrial field. The vector control of a PMSM requires electrical rotor speed and position. However, position sensors require additional space and increase cost. So, many researchers have studied sensorless controls of the PMSM, and several methods such as state observer, adaptive observer and sliding-mode observer on backelectromotive force have been developed [1-4].

A stochastic method using an extended Kalman filter (EKF) is one of sensorless control methods. The EKF observer can provide the good estimation of state values through a Kalman gain and recursive state values [5-10].

Recently, Julier and Uhlman proposed a new scheme using Kalman filter with a mapping transformation method such as an unscented transformation (UT) instead of a linearization process of the EKF. It is called the unscented Kalman filter (UKF). The linearization process often involves error between the linearized model and the system model [10-11]. The UKF observer has previously been introduced [12-15]. Ref. 12 compared two Kalman filters. The application of the UKF observer was reported [13]. High-speed startup [14] and load-torque observation [15] have been applied to a motor drive.

There are two kinds of the UT methods: basic UT (BUT)

$\dagger$ Corresponding Author: Dept. of Electrical and Computer Engineering, Pusan National University, Korea. (yakwon@pusan.ac.kr)

* Dept. of Electrical and Computer Engineering, Pusan National University, Korea. (mch99100@pusan.or.kr)

Received: November 17, 2014; Accepted: September 25, 2015 and general UT (GUT) [11]. The BUT and GUT can be distinguished by scaling parameter value. Resultantly, the weight, sample number and computation time are also different. Sensorless control has been usually based on the BUT because of the scaling parameter. The researchers have trouble in selecting a proper scaling parameter value and it is not obvious. Ref. 15 introduced the GUT observer for a direct torque control using the scaling parameter of 1 .

In this paper, the UKF observer with a variety of scaling parameter is investigated and compared. This observer has been applied to the sensorless speed control of a PMSM, and has been performed under the forward and reverse rotation, and the load variation.

The unscented transformation is divided as follow according to the scaling parameter value.

1) $\kappa=0$, this case is called the BUT without a zeroth sigma point and weight.

2) $\kappa \neq 0$, this case is called the GUT with zeroth sigma point and weight.

3) $\kappa=0.5$, the zeroth weight and other weights are same values.

4) $\kappa>0.5$, the zeroth weight is bigger than other weights.

\section{Mathematical Model of the PMSM}

To control the AC motor, a coordinate transformation is used to reduce the complexity of the three-phase voltage equations. A mathematical modeling of the PMSM for sensorless control needs to take into account the followings: the voltage equations in the $\alpha-\beta$ stationary reference frame, and the electrical rotor position and speed. The nonlinear 
state equations are as follows:

$$
\begin{gathered}
\mathbf{x}_{k+1}=\mathbf{f}\left(\mathbf{x}_{k}\right)+\mathbf{B} \mathbf{v}_{k}+\boldsymbol{\omega}_{k}, \\
\mathbf{y}_{k+1}=\mathbf{h}\left(\mathbf{x}_{k}\right)+\mathbf{v}_{k} .
\end{gathered}
$$

The state matrix consists of four state variables, $x=\left[i_{\alpha s} i_{\beta S} \omega_{r} \theta_{r}\right]^{T}$. The input and output matrices are $v=\left[v_{\alpha s_{-} r e f} v_{\beta s_{-} r e f}\right]^{T}$ and $\mathbf{y}=\left[i_{\alpha s} i_{\beta s}\right]^{T}$, respectively. $v_{\alpha \beta s}$ and $i_{\alpha \beta s}$ are the stator voltages and currents in the $\alpha-\beta$ stationary reference frame, respectively. $\omega_{r}$ and $\theta_{r}$ are the electrical rotor speed and electrical rotor position. $\boldsymbol{\omega}_{k}$ is defined as the system noise that affects the model, and $\boldsymbol{v}_{k}$ is defined as the measurement noise of the sensor. It is generally assumed that the system and measurement noises are white Gaussian noises.

If the electrical speed is assumed to be constant during the sampling period, then the differential value can be regarded as zero. The state matrix of the UKF observer, input matrix, and output matrix are as follows:

$$
\begin{aligned}
& \mathbf{f}(\mathbf{x})=\left[\begin{array}{c}
f_{1} \\
f_{2} \\
f_{3} \\
f_{4}
\end{array}\right]=\frac{1}{L_{s}}\left[\begin{array}{c}
-R_{s} i_{\alpha}+\Psi_{m} \omega_{r} \sin \left(\theta_{r}\right) \\
-R_{s} i_{\beta}-\Psi_{m} \omega_{r} \cos \left(\theta_{r}\right) \\
0 \\
L_{s} \omega_{r}
\end{array}\right], \\
& \mathbf{B}=\frac{1}{L_{s}}\left[\begin{array}{llll}
1 & 0 & 0 & 0 \\
0 & 1 & 0 & 0
\end{array}\right]^{T}, \mathbf{h}(\mathbf{x})=\left[\begin{array}{l}
h_{1} \\
h_{2}
\end{array}\right]=\left[\begin{array}{l}
i_{\alpha} \\
i_{\beta}
\end{array}\right] \text {. }
\end{aligned}
$$

where, $\mathrm{L}_{\mathrm{s}}$ is the stator inductance, $R_{s}$ is the stator resistance, and $\psi_{m}$ is the flux linkage of the permanent magnet.

\section{Unscented Transformation and Unscented Kalman Filter}

\subsection{Unscented transformation}

The accuracy of the conversion process is proportional to the number of samples. However, increasing the number of samples leads to the algorithm's complexity. The aim of the UT is to obtain the mean and covariance. In the UT, the sigma point $\chi$ is calculated from mean and factorization of the covariance. The weight $W$ is a constant that determines the specific gravity of the estimated values.

The distribution of the each estimated values can be specified by scaling parameter $\kappa$. First, the lower triangular matrix $\boldsymbol{u}$ using the Cholesky factorization is calculated. Then, the sigma points and weights are determined. The sigma points and weights are determined as follows.

$$
\mathbf{U}^{T} \mathbf{U}=(n+\kappa) \mathbf{P}_{x} .
$$

$$
\begin{gathered}
\chi_{1}=\mathbf{x}, \quad \chi_{i+1}=\mathbf{x}+\mathbf{u}_{i}, \quad \chi_{i+n+1}=\mathbf{x}-\mathbf{u}_{i}, \\
i=1,2, \ldots, n . \\
W_{1}=\frac{\kappa}{n+\kappa}, \quad W_{i+1}=\frac{1}{2(n+\kappa)}, \\
W_{i+n+1}=\frac{1}{2(n+\kappa)}, \quad i=1,2, \ldots, n
\end{gathered}
$$

where, $n$ is the number of state variable, $\mathbf{x}$ is the state variable matrix, $\mathbf{P}_{\mathrm{x}}$ is the covariance matrix, and a vector element $\mathbf{u}_{i}$ is a row vector of the matrix $\mathbf{U}$. Additionally, the sum of weight is always 1 .

As mentioned earlier, the scaling parameter $\kappa$ in the BUT is 0 , and the zeroth sigma point and weight are removed. The zeroth sigma point means the estimated average value or standard point. If the number of state variable is equal to $n$, the number of sigma points is $2 * n^{2}$. Each of weight values are always a uniform value relying on $1 /(2 * n)$. In the GUT, the number of sigma points is $n *(2 * n+1)$. It is possible to ensure relatively high precision because of the increasing number of sigma points, but the disadvantage is that computation time also increases.

\subsection{Unscented Kalman filter}

The UKF uses a unique method based on a nonlinear transformation instead of a linearization process of EKF. This method makes an elaborate choice for each sample using a sigma point and weight which is yielded form the state and covariance values. The UKF algorithm has a little complexity, but the overall process remains unchanged namely performing a prediction, Kalman gain and estimation step. Using the previously estimated state, the prediction step calculates the expected state and error covariance values. Next step calculates the Kalman gain. Last, the update step estimates the error covariance values and the exact status on the basis of the relation between the previously calculated prediction state values and the actually measured state values. The three steps are the same as the EKF. The overall procedure is as follows [10].

1) Initialization

2) Calculation of sigma points and weights.

3) Prediction of the estimation value and error covariance.

$$
\begin{gathered}
\hat{\mathbf{x}}_{k \mid k-1}=\sum_{i=1}^{2 n+1} W_{i} \mathbf{f}\left(\chi_{i}\right) \\
\mathbf{P}_{x, k \mid k-1}=\sum_{i=1}^{2 n+1} W_{i}\left(\mathbf{f}\left(\chi_{i}\right)-\hat{\mathbf{x}}_{k \mid k-1}\right)\left(\mathbf{f}\left(\chi_{i}\right)-\hat{\mathbf{x}}_{k \mid k-1}\right)^{T}+\mathbf{Q} .
\end{gathered}
$$

4) Prediction of the measurement value and error covariance.

$$
\hat{\mathbf{y}}_{k \mid k-1}=\sum_{i=1}^{2 n+1} W_{i} \mathbf{h}\left(\chi_{i}\right) .
$$


$\mathbf{P}_{y, k \mid k-1}=\sum_{i=1}^{2 n+1} W_{i}\left(\mathbf{h}\left(\boldsymbol{\chi}_{i}\right)-\hat{\mathbf{y}}_{k \mid k-1}\right)\left(\mathbf{h}\left(\boldsymbol{\chi}_{i}\right)-\hat{\mathbf{y}}_{k \mid k-1}\right)^{\mathrm{T}}+\mathbf{R}$.

5) Calculation of the Kalman gain.

$$
\begin{gathered}
\mathbf{P}_{x y, k \mid k-1}=\sum_{i=1}^{2 n+1} W_{i}\left(\mathbf{f}\left(\boldsymbol{\chi}_{i}\right)-\hat{\mathbf{x}}_{k \mid k-1}\right)\left(\mathbf{h}\left(\boldsymbol{\chi}_{i}\right)-\hat{\mathbf{y}}_{k \mid k-1}\right)^{\mathrm{T}} . \\
\mathbf{K}_{k}=\mathbf{P}_{\mathrm{xy}} \mathbf{P}_{y}^{-1} .
\end{gathered}
$$

6) Correction of the estimation and the covariance.

$$
\begin{gathered}
\hat{\mathbf{x}}_{k}=\hat{\mathbf{x}}_{k \mid k-1}+\mathbf{K}_{\mathrm{k}}\left(\mathbf{y}_{k}-\hat{\mathbf{y}}_{\mathrm{k}}\right) . \\
\mathbf{P}_{k}=\mathbf{P}_{k \mid k-1}-\mathbf{K}_{\mathrm{k}} \mathbf{P}_{y} \mathbf{K}_{k}^{T} .
\end{gathered}
$$

\section{Experimentation and Discussion}

To verify the performance of UKF observer, the experiments have been carried out. The overall system is shown in Fig. 1. The speed and current controllers have serial connection in the control system. This motor runs according to the speed reference value at zero point.

The experiments have been carried out for the sensorless speed control of a PMSM with 8 poles and $1 \mathrm{hp}$. The controller is using TMS320F28335 (150MHz). Fig. 2 shows the experimental set-up. The sampling time is $1 \mathrm{~ms}$ for speed control, and $200 \mu \mathrm{s}$ for current control. The

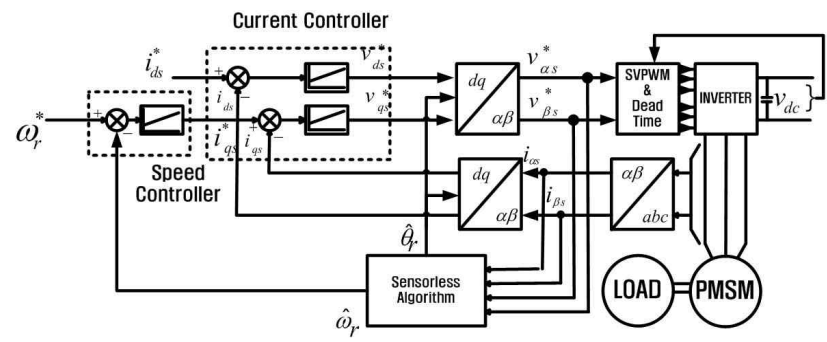

Fig. 1. Block diagram of overall system

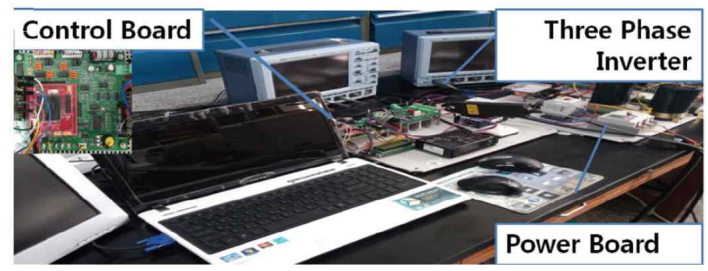

(a)

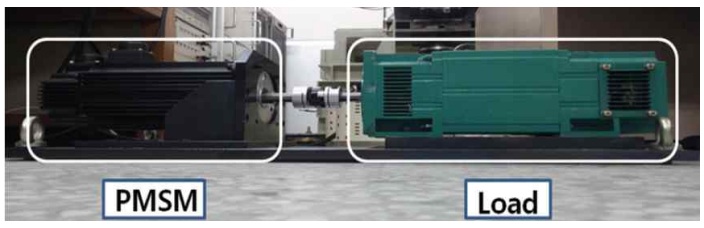

(b)

Fig. 2. Construction of experimental equipments.
PMSM is equipped with an incremental encoder of 2000 PPR, and it is used for the comparison of the estimated and actual positions. The load is a DC motor of $750 \mathrm{~W}$. Several waveforms are observed by oscilloscopes, and the state of the motor control is analyzed in this study.

The parameters of a PMSM used in the experiment are presented in Table 1.

Table 1. PMSM parameters

\begin{tabular}{c|c}
\hline Stator resistance & $1.5 \Omega$ \\
Stator inductance & $4.87 \mathrm{mH}$ \\
Number of poles & 8 \\
Rated power & $750 \mathrm{w}$ \\
Rated Current & $5.3 \mathrm{~A}$ \\
Rated Speed & $2000 \mathrm{rpm}$ \\
Rated torque & $3.58 \mathrm{Nm}$ \\
\hline
\end{tabular}

The code size and computation time for the different UT methods are summarized in Table 2. As a result, the advantage of basic UT has the shortest computation times There is $12.92 \mu$ s difference between the BUT and GUT. This difference relies on the fact of existence or nonexistence of the scaling parameter.

Table 2. Comparison of code size and computation times

\begin{tabular}{c|c|c}
\hline & Code size (byte) & Computation time $(\mu \mathrm{s})$ \\
\hline BUT & 11,627 & 75.34 \\
GUT & 12,219 & 88.26 \\
\hline
\end{tabular}

Table 3. Comparison of weight according to the scaling parameter

\begin{tabular}{c|c|c|c}
\hline & $\begin{array}{c}\text { Scaling } \\
\text { parameter }\end{array}$ & Weights & Sum of weights \\
\hline $\begin{array}{c}\text { Basic } \\
\text { UT }\end{array}$ & 0 & $\begin{array}{c}\mathrm{W}_{1}=0, \\
\mathrm{~W}_{2 \sim 9}=1 / 8=0.1250\end{array}$ & $\mathrm{~W}_{\text {total }}=0+1 / 8 * 8=1$ \\
\hline & 0.5 & $\begin{array}{c}\mathrm{W}_{1}=1 / 9=0.111, \\
\mathrm{~W}_{2 \sim 9}=1 / 9=0.1111\end{array}$ & $\mathrm{~W}_{\text {total }}=1 / 9+1 / 9 * 8=1$ \\
General & 1 & $\begin{array}{c}\mathrm{W}_{1}=2 / 10=0.200, \\
\mathrm{~W}_{2 \sim 9}=1 / 10=0.1000 \\
\mathrm{~W}_{1}=32 / 40=0.800, \\
\mathrm{~W}_{2 \sim 9}=1 / 40=0.0250 \\
\mathrm{~W}_{1}=72 / 80=0.900, \\
\mathrm{~W}_{2 \sim 9}=1 / 80=0.0125\end{array}$ & $\mathrm{~W}_{\text {total }}=2 / 10+8 / 10 * 8=1$ \\
& 16 & $\mathrm{~W}_{\text {total }}=32 / 40+1 / 40 * 8=1$ \\
\end{tabular}

Table 3 lists the weight change according to the scaling parameter. From the results described above, if the scaling parameter is more than 0.5 , the value of the zeroth weight is higher than the other ones.

To illustrate scaling parameter performance in highspeed operation, the motor runs at zero, increases to the rated speed of $2000 \mathrm{rpm}$ for $2 \mathrm{sec}$, and then reverse to the backward rated speed of $-2000 \mathrm{rpm}$. We compared the performance of the state estimation in each case. Initial transient state time is $100 \mathrm{~ms}$, and transient state time is $200 \mathrm{~ms}$ on forward and reverse rotation. The experimental results for the BUT $(\kappa=0)$, GUT-I $(\kappa=1)$, and GUT-II $(\kappa=36)$ are shown in Fig. 3(a), (b) and (c), respectively. It shows the good speed estimation regardless of the scaling 

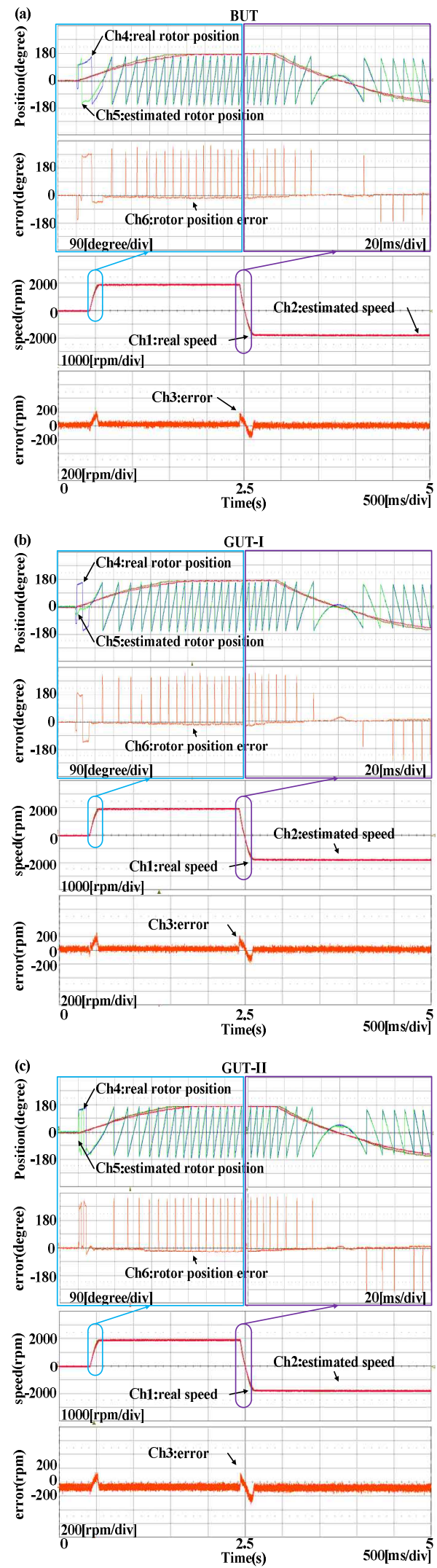

Fig. 3. Position and speed responses in the high speed $(2000 \rightarrow-2000 \mathrm{rpm}): \quad(\mathrm{a}) \mathrm{BUT}(\kappa=0)$, (b) GUT-I $(\kappa=1),(\mathrm{c})$ GUT-II $(\kappa=36)$
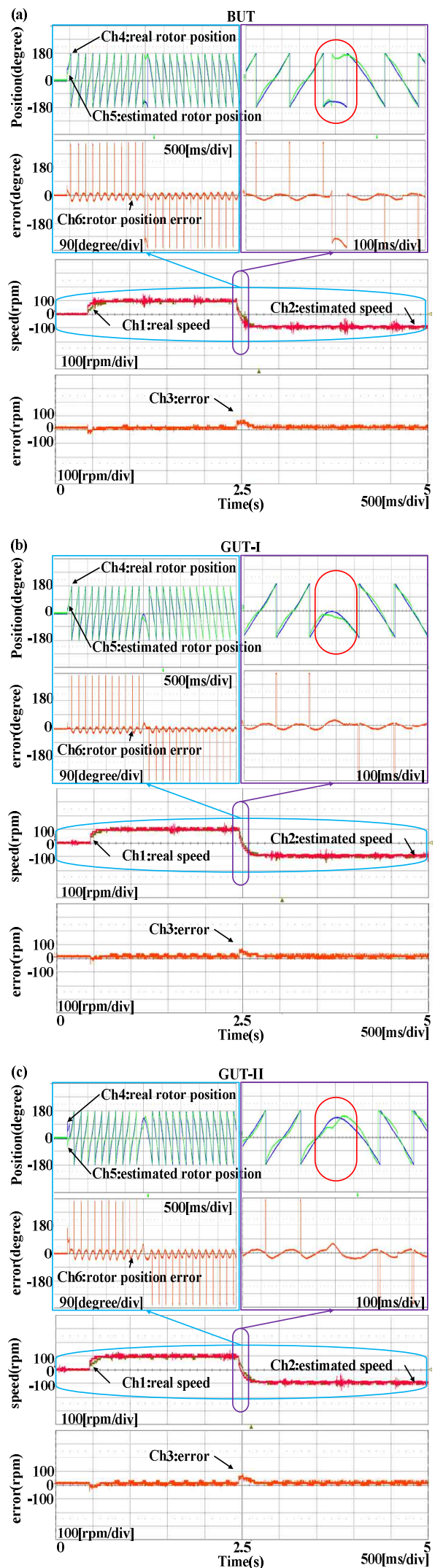

Fig. 4. Position and speed responses in the low speed (100 $\rightarrow-100 \mathrm{rpm})$ : (a) BUT $(\kappa=0)$, (b) GUT-I $(\kappa=1)$, (c) GUT-II $(\kappa=36)$ 

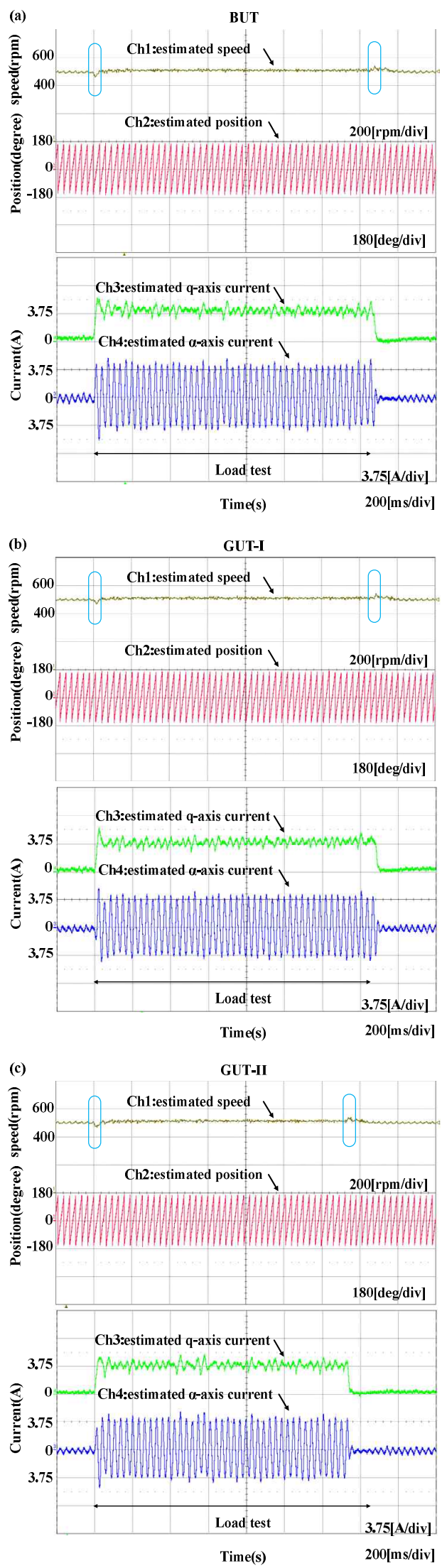

Fig. 5. Speed responses and current waveforms in the load variation $(500 \mathrm{rpm}, 0 \rightarrow 75 \% \rightarrow 0$ load): (a) BUT $(\kappa=0),(b)$ GUT-I $(\kappa=1),(c)$ GUT-II $(\kappa=36)$ parameter, but does not meet the initial position estimation. In the case of BUT, the initial position estimation value is unstable for $28 \mathrm{~ms}$, but it shows outstanding estimation values at near the zero point. In the case of GUT-I and -II, the results show that the initial estimated position has been unstable for $16 \mathrm{~ms}$. It indicates that the GUT has relatively better than the BUT. But, in the near zero point, estimated position has a little error for $16 \mathrm{~ms}$.

Fig. 4 shows the performances in the low speed from 100 to $-100 \mathrm{rpm}(0.05 \%$ of rated speed $)$ in the sensorless speed control using a UKF observer with a variety of UT methods. Fig. 4 (a), (b) and (c) present the result of the position and speed estimations in the BUT, GUT-I and GUT-II, respectively. There is some difference between the actual and estimated speeds in the transient state, however, it converges quickly. In the case of GUT-I, the UKF observer for the electrical rotor position has excellent performance. In the case of GUT-II, it has $37 \mathrm{rpm}$ maximum error than other methods in transient state. The BUT method has good performance. In the case of reverse rotation, the experimental result shows that the estimated position has a considerable error in the near zero point.

The performances of various UT methods are illustrated in Fig. 5 when the load of $2.7 \mathrm{Nm}(=75 \%)$ is applied and is removed. The estimated speed is decreased in a flash when the load torque was applied, and is increased immediately when the load is removed. But both estimated speeds are quickly converged to the reference speed. It can prove that the sensorless observers are robust in rejecting a disturbance regardless of scaling parameter value.

The BUT observer shows a good estimation in a wide speed range. The GUT-I $(\kappa=1)$ has a quick speed response in transient state in case of a low speed and the estimated electrical rotor position has relatively excellent performance. At high start-up speed, the estimated electrical position has the unstable chattering in a moment, if the scaling parameter is very large. In the case of BUT, in the initial transient state, it needs a little more time to stabilize the position, relatively. As a result, it can be seen that the GUT-I is the most ideal performance. We found no significant difference between BUT and GUT in the load variation under a constant speed. In the computation times, it was found that the BUT results in a reduction of approximately $13 \mu \mathrm{s}$.

\section{Conclusion}

A new sensorless control of a permanent magnet synchronous motor using the UKF has been suggested in this paper. Several key advantages and disadvantages on the UT methods having different scaling parameter values are shown in the result. The performance of forward and reverse rotations in the low and high speeds, and the disturbance performance using the BUT and GUT are investigated in this study, where the GUT has the scaling 
parameters of 1 and 36. Additionally, the code size and the computation time are compared. As a result, the UKF method is satisfactory enough to implement a sensorless control of a permanent magnet synchronous motor over a wide speed range in terms of a state observer. Also, the UKF observer is robust in rejecting a disturbance regardless of scaling parameter value.

\section{Acknowledgement}

This work was supported by a 2-Year Research Grant of Pusan National University.

\section{References}

[1] B. K. Bose, "Technology trends in microprocessor control of electrical machines," IEEE Trans. Ind. Elec., vol. 35, no. 1, pp. 160-177, Feb. 1988.

[2] K. Kamiyama, T. Ohmae, and T. Sukegawa, "Application trends in AC motor drives," in proc. IECON'92, San Diego, CA, vol. 1, pp. 31-36, 1992.

[3] H. Lin, K-Y. Hwang, and B-I. Kwon, "An Improved Flux Observer for Sensorless Permanent Magnet Synchronous Motor Drives with Parameter Identification," Journal of Electrical Engineering \& Technology, vol. 8, no. 3, pp. 516-523, May 2013.

[4] J. S. Kim, and S. K. Sul, "New approach for highperformance PMSM drives without rotational position sensors," IEEE Trans. Power Electron., vol. 12, no. 5, pp. 904-911, Sep. 1997.

[5] R. E. Kalman, "A new approach to linear filtering and prediction problems," Trans. ASME J. Basic Eng., vol. 82, no. 1, pp. 35-45, Mar. 1960.

[6] H-W. Sim, J-S. Lee, and K-B. Lee, "On-line parameter estimation of interior permanent magnet synchronous motor using an extended Kalman filter," Journal of Electrical Engineering \& Technology, vol. 9, no. 2, pp. 600-608, March 2014.

[7] S. Bolognani, R. Oboe, and M. Zigliotto, "Sensorless full-digital PMSM drive with EKF estimation of speed and rotor position," IEEE Trans. Ind. Elec., vol. 46, no. 1, pp. 184-191, Feb. 1999.

[8] S. Bolognani, M. Zigliotti, M. Zordan, "ExtendedRange PMSM Sensorless Speed Drive Based on Stochastic Filtering," IEEE Trans. Power Electronics, vol. 16, no. 1, pp. 110-117, Jan. 2001.

[9] H-W. Kim and S-K. Sul, "A new motor speed estimator using Kalmna filter in low-speed range," IEEE Trans. Ind. Elec., vol. 43, no. 4, pp. 498-504, Aug. 1996.

[10] S. J. Julier and J. K. Uhlmann, "A new extension of the Kalman filter to nonlinear systems," in Proc. AeroSense: 11th Int. Symp. Aerospace/Defense Sensing,
Simulations and Controls, pp. 182-193, 1997.

[11] S. Jafarzadeh, C. Lascu, and M. Fadali, "State estimation of induction motor drives using the unscented Kalman filter," IEEE Trans. Ind. Electron., vol. 59, no. 11. pp. 4207-4216., Nov. 2012.

[12] P. Borsje, T. F. Chan, Y. K. Wong, and S. L. Ho, “A comparative study of Kalman filtering for sensorless control of a permanent-magnet synchronous motor drive," in IEEE Int. Conf. Electr. Machines Drives, San Antonio, TX, pp. 815-822, 2005.

[13] T.-F. Chna, P. Borsje, and W. Wang "Application of unscented Kalman filter to sensorless permanentmagnet synchronous motor drive," in IEEE Int. Conf. Electr. Machines Drives, Miami, FL, pp.631-638, 2009.

[14] A. Cichowski, S. Bujacz, J. Nieznanski, and P. Szczepankowski, "Sensorless startup of super high speed permanent magnet motor," IEEE Int. Symp. Ind Electron., Bari, pp. 3101-3106 , 2010.

[15] D. Janiszewski, "Load torque estimation in sensorless PMSM drive using unscented Kalman filter," IEEE Int. Symp. Ind. Electron., Gdansk, pp. 643-648, 2011.

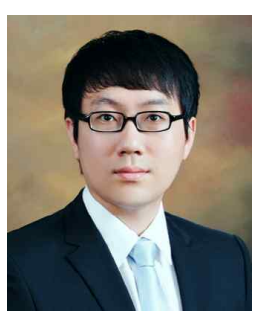

Cheol Moon received B.S and M.S degree in electrical engineering from Pusan National University, Busan, Korea in 2010 and 2012, respectively. $\mathrm{He}$ is currently working toward the $\mathrm{Ph} . \mathrm{D}$. degree. His research interests include power electronics and electric machine control.

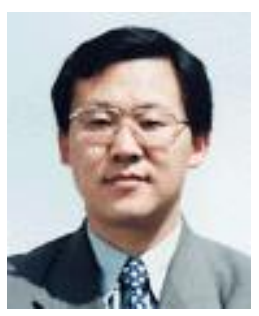

Young-Ahn Kwon received B.S, M.S, and $\mathrm{Ph} . \mathrm{D}$. degree in electrical engineering from Seoul National University, Seoul, Korea in 1978, 1983, and 1986, respectively. He is currently a Professor in the Department of Electrical and Computer Engineering, Pusan National University, Busan, Korea. His research interests include electric machines, power electronics, and control. 